\title{
Identification of QTLs for Blast, Bacterial Blight, and Planthopper Resistance Using SNP-Based Linkage Maps from Two Recombinant Inbred Rice Lines
}

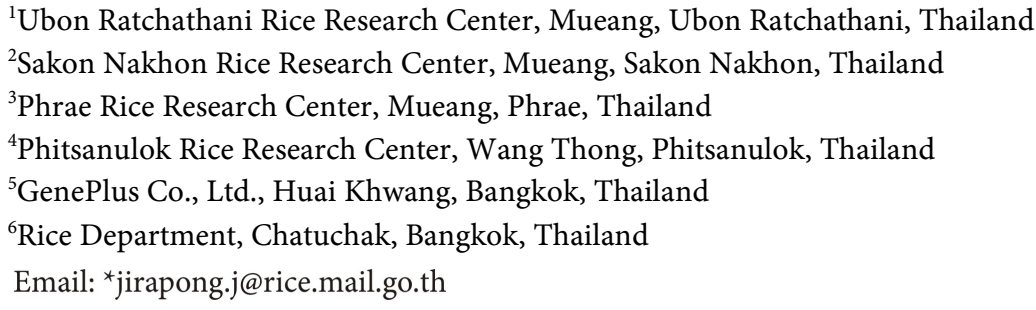

Jirapong Jairin ${ }^{*}$, Phanchita Vejchasarn11, Thanapa Somjai' ${ }^{1}$, Kanuengnij Srivilai ${ }^{2}$ Kulchana Darwell ${ }^{3}$, Phikul Leelagud1, Rungnapa Kawichai', Jate Kotcharerk ${ }^{4}$, Arissara Suthanthangjai1, Nattaya Popa ${ }^{5}$, Suphalaksana Lachanthuek ${ }^{6}$, Varapong Chamarerk ${ }^{6}$

How to cite this paper: Jairin, J., Vejchasarn, P., Somjai, T., Srivilai, K., Darwell, K., Leelagud, P., Kawichai, R., Kotcharerk, J., Suthanthangjai, A., Popa, N., Lachanthuek, S. and Chamarerk, V. (2019) Identification of QTLs for Blast, Bacterial Blight, and Planthopper Resistance Using SNP-Based Linkage Maps from Two Recombinant Inbred Rice Lines. American Journal of Plant Sciences, 10, 760-779.

https://doi.org/10.4236/ajps.2019.105056

Received: March 26, 2019

Accepted: May 24, 2019

Published: May 27, 2019

Copyright $\odot 2019$ by author(s) and Scientific Research Publishing Inc. This work is licensed under the Creative Commons Attribution International License (CC BY 4.0).

http://creativecommons.org/licenses/by/4.0/

\begin{abstract}
Rice is the most significant global food security. Several biotic factors limit rice production, breeding biotic-resistant rice has, therefore, become an increasingly important goal. Two elite rice lines, IR71033-121-15 (IR71033) and IR57514-PMI-5-B-1-2 (IR57514), provide potential genes for biotic stress resistance traits. In this study, genotyping by sequencing (GBS) for single nucleotide polymorphism (SNP)-based linkage map construction was used to detect quantitative trait loci (QTLs) for blast (BL), bacterial blight (BB), whitebacked planthopper (WBPH), and brown planthopper (BPH) resistance. IR71033 was derived from Oryza minuta and carried BL, BB, WBPH, and BPH resistance QTLs. IR57514 is a well-adapted rainfed lowland line that carries BL and BB resistance QTLs. Two sets of recombinant inbred line (RIL) populations derived from crosses of KDML105 $\times$ IR71033 and KDML105 $\times$ IR57514 were used to dissect the genetic basis of disease and insect pest resistance. The RIL populations were evaluated for BL, BB, WBPH, and BPH resistance from 2016 to 2018 at four rice research centers in Thailand. From these, we identified a large number of SNPs through GBS and constructed high-resolution linkage maps. By combining phenotypic evaluation with the GBS data, a total of 24 QTLs on four chromosomes were detected that confered pest resistance and explained $7.3 \%-61.4 \%$ of the pheno-
\end{abstract}


typic variance. These findings should facilitate identifying novel resistance genes and applying marker-assisted selection for resistance to the four major rice pests investigated here. These strategies will improve the resilience and reliability of rice varieties adapted to the low-yielding environment of rainfed lowland areas worldwide.

\section{Keywords}

Recombinant Inbred Line, Genotyping by Sequencing, Resistance Gene, Rainfed Lowland Rice, Single Nucleotide Polymorphism, Quantitative Trait Locus

\section{Introduction}

Among the biotic stressors of rice, blast (BL), bacterial blight (BB), white backed planthopper (WBPH), and brown planthopper (BPH) are the four major pathogens affecting rainfed lowland rice; they are caused by Magnaporthe grisea, Xanthomonas oryzae pv. oryzae, Sogatella furcifera, and Nilaparvata lugens, respectively. These destructive pests greatly reduce rice yield throughout the rainfed lowlands in Asia and also in the northeast (NE) of Thailand [1] [2]. The NE region accounts for over half of Thailand's total rice production area [3] and is well known as a major producer of premium Jasmine rice "Khao Dawk Mali 105 " (KDML105). Approximately $65 \%$ of the area is dominated by KDML105, which is well adapted to the low-yielding environment of rainfed lowland conditions [2]. However, this strain is highly susceptible to $\mathrm{BL}, \mathrm{BB}, \mathrm{WBPH}$, and $\mathrm{BPH}$. In 2018, BL and BPH outbreaks destroyed close to $80 \%$ of the rice crop in the rainfed lowland areas of the lower part of the NE [4]. Therefore, the development of resistant varieties is considered to be the most effective and economical means of maintaining yield stability by controlling the major rice pests in the frequent outbreak areas [5] [6].

To date, the various locations on the rice chromosomes of $\mathrm{BL}, \mathrm{BB}, \mathrm{WBPH}$, and $\mathrm{BPH}$ resistance genes have been reported. More than one-hundred major resistance genes and over 350 quantitative trait loci (QTLs) for BL have been identified in the rice genome [7] [8] and, among them, 30 genes have already been cloned [9]. About 44 major genes conferring resistance to $\mathrm{BB}$ have been identified in cultivated rice, wild relatives, and mutation-induced lines [10] [11] [12]. At least 34 major genes have been identified for resistance against planthoppers from wild and cultivated rice germplasm [13]. Taken together, this signifies that recent advances in rice genomic research have enabled the rapid identification of various rice pest resistance genes/QTLs and provided DNA markers for marker-assisted selection (MAS). MAS has been used to transfer and pyramid genes/QTLs associated with biotic stressors into KDML105. Numerous promising lines and varieties that maintain the cooking quality and fragrance of KDML105 have been developed [2] [14]. These improved varieties have been re- 
leased in the target area in NE Thailand [1]. However, varieties containing a major resistance gene induce the rapid evolution of new biotypes of pathogens [15] [16]. The continuous evolution of pathogenic biotypes causes the breakdown of resistance in many improved varieties. Therefore, novel resistance genes are still highly in demand for continued rice improvement and more analyses of resistance genes are needed to combat destructive pests going forward.

The present study uses two elite rice lines, IR71033-121-15 (IR71033) and IR57514-PMI-5-B-1-2 (IR57514), from the International Rice Research Institute. The introgression line IR71033, derived from an interspecific cross between IR31917-45-3-2 and a wild species Oryza minuta, has been intensively used as a donor for biotic stress resistance studies [17] [18] [19] [20]. The elite line IR57514, derived from a three-way cross of IR43581-57-3-3-6/KDML105// IR21836-90-3, is well adapted to the low-yielding rainfed lowland environment in the NE of Thailand [21] [22]. IR71033 is resistant to BL, BB, WBPH, and BPH and IR57514 is resistant to BL and BB. These two rice lines were selected as donors for biotic resistance in breeding programs for improving rice cultivation in rainfed lowland area in NE. To identify biotic stress resistance factors in both rice lines, two recombinant inbred lines (RILs) were developed and used as mapping populations.

In the present study, next-generation sequencing (NGS) technology was used to discover single nucleotide polymorphism (SNP). NGS has reduced both the cost and the time required to generate sequence data. For SNP identification, NGS methods were combined with restriction enzyme digest to reduce genome complexity and to enable cost efficiency [23]. These data were then used to generate SNP-based linkage maps. The objective of this study was to identify loci associated with BL, BB, WBPH, and BPH resistance by QTL analysis using SNP marker sets. The tightly-linked SNP markers will facilitate MAS, and the investigated rice lines can serve as genetic resources for $\mathrm{BL}, \mathrm{BB}, \mathrm{WBPH}$, and $\mathrm{BPH}$ resistance studies to strengthen the resilience of rice varieties and improve breeding programs, especially in rainfed lowland regions.

\section{Materials and Methods}

\subsection{Plant Materials and Mapping Populations}

Two RIL populations derived from crosses of KDML105 × IR71033-121-15 (286 lines) and KDML105 $\times$ IR57514-PMI-5-B-1-2 (288 lines) were used as the mapping population. KDML105 is susceptible to BL, BB, WBPH, and BPH. IR71033 is resistant to $\mathrm{BL}, \mathrm{BB}, \mathrm{WBPH}$, and $\mathrm{BPH}$. IR57514 is resistant to $\mathrm{BL}$ and $\mathrm{BB}$. The cross KDML105 × IR71033 (hereafter called "KD-IR71033") was developed at the Ubon Ratchathani Rice Research Center (rice lines were designated as UBN04128), while the cross KDML105 × IR57514-PMI-5-B-1-2 (hereafter called "KD-IR57514") was developed at the Phitsanulok Rice Research Center (rice lines were designated as PSL99093). Rice cultivars, TN1, KDML105, KTH17, RD10, PSL2, Rathu Heenati, PTB33, HY71, SPR1, RD7, IRBB5, and IRBB7, were 
used as resistant and susceptible controls for resistance evaluations (Table 1).

\subsection{Phenotypic Evaluations}

\subsubsection{Field BL Nursery Evaluation}

The upland BL nurseries were set up in the wet season at three rice research centers: Ubon Ratchathani Rice Research Center (UBN-RRC) in 2016 and 2018, Sakon Nakhon Rice Research Center (SKN-RRC) in 2016 and 2018, and Phrae Rice Research Center (PRE-RRC) in 2016 (Table 2, Figure 1). Seeds for 286 and 288 RILs from KD-IR71033 and KD-IR57514, respectively, were planted in single-row plots, $60 \mathrm{~cm}$ in length, in BL nursery beds. Every five entries were separated by a susceptible check (KDML105 or KTH17) and resistance check (SPR1 or HY71). KDML105 was planted alongside the nursery bed in spreader rows to enhance the spore transmission. Plots and spreader rows were inoculated with a local natural mix of strains. Standard Evaluation System (SES) ratings for BL [24] were used when all of the susceptible control rows died.

Table 1. Details of rice varieties used as resistant and susceptible controls for the phenotypic evaluations.

\begin{tabular}{|c|c|c|}
\hline Rice variety & Origin & Description \\
\hline TN1 & Taiwan & $\begin{array}{l}\text { The inbred rice variety was used as susceptible control for BPH, } \\
\text { WBPH and BB at UBN-RRC and PSL-RRC. }\end{array}$ \\
\hline KDML105 & Thailand & $\begin{array}{l}\text { The Thai rice landrace was used as susceptible control for BPH, } \\
\text { WBPH, BB and BL at UBN-RRC, PRE-RRC, SKN-RRC and PSL-RRC. }\end{array}$ \\
\hline Rathu Heenati & Sri Lanka & $\begin{array}{l}\text { The Sri Lankan rice landrace was used as resistant control for BPH at } \\
\text { UBN-RRC and PSL-RRC. }\end{array}$ \\
\hline РTB33 & India & $\begin{array}{l}\text { The Indian rice landrace was used as resistant control for WBPH at } \\
\text { UBN-RRC and PSL-RRC. }\end{array}$ \\
\hline KTH17 & Thailand & $\begin{array}{l}\text { The Thai rice landrace was used as susceptible control for BL at } \\
\text { PRE-RRC. }\end{array}$ \\
\hline HY71 & Thailand & $\begin{array}{l}\text { The Thai rice landrace was used as resistant control for BL at } \\
\text { PRE-RRC. }\end{array}$ \\
\hline SPR1 & Thailand & $\begin{array}{l}\text { The inbred rice variety was used as resistant control for BL at } \\
\text { UBN-RRC. }\end{array}$ \\
\hline $\mathrm{RD} 10$ & Thailand & $\begin{array}{l}\text { The inbred rice variety was used as susceptible control for BB at } \\
\text { PRE-RRC. }\end{array}$ \\
\hline PSL2 & Thailand & $\begin{array}{l}\text { The inbred rice variety was used as susceptible control for BB at } \\
\text { PRE-RRC. }\end{array}$ \\
\hline RD7 & Thailand & $\begin{array}{l}\text { The inbred rice variety was used as resistant control for BB at } \\
\text { PRE-RRC. }\end{array}$ \\
\hline IRBB5 & IRRI & $\begin{array}{l}\text { The inbred rice variety was used as resistant control for BB at } \\
\text { PRE-RRC. }\end{array}$ \\
\hline IRBB7 & IRRI & $\begin{array}{l}\text { The inbred rice variety was used as resistant control for BB at } \\
\text { PRE-RRC. }\end{array}$ \\
\hline
\end{tabular}


Table 2. Phenotypic trait and resistance evaluations for the two RILs populations, KD-IR71033 and KD-IR57514 at Rice Research Centers in Thailand.

\begin{tabular}{cccccc}
\hline Location & Region & Ecosystem & 2016 & 2017 & 2018 \\
\hline KD-IR71033 & & & & & \\
\hline UBN-RRC & Northeast & Rainfed & $\mathrm{BL}^{\mathrm{DS}}, \mathrm{BB}^{\mathrm{LL}}, \mathrm{BPH}^{\mathrm{DS}}$ & $\mathrm{BB}^{\mathrm{DS}}$ & $\mathrm{BL}^{\mathrm{DS}}$ \\
SKN-RRC & Northeast & Rainfed & $\mathrm{BL}^{\mathrm{DS}}, \mathrm{BB}^{\mathrm{DS}}$ & - & - \\
UDN-RRC & Northeast & Rainfed & $\mathrm{BB}^{\mathrm{DS}}$ & - & - \\
PRE-RRC & North & Irrigated & $\mathrm{BL}^{\mathrm{DS}}$ & - & - \\
PSL-RRC & North & Irrigated & - & - & $\mathrm{WBPH}^{\mathrm{DS}}$ \\
KD-IR57514 & & & & & \\
UBN-RRC & Northeast & Rainfed & - & - & $\mathrm{BL}^{\mathrm{DS}}$ \\
SKN-RRC & Northeast & Rainfed & - & - & $\mathrm{BL}^{\mathrm{DS}}, \mathrm{BB}^{\mathrm{LL}}$ \\
UDN-RRC & Northeast & Rainfed & - & - & $\mathrm{BB}^{\mathrm{LL}}$ \\
\hline
\end{tabular}

DS based on damage score, LL based on lesion leaf length.

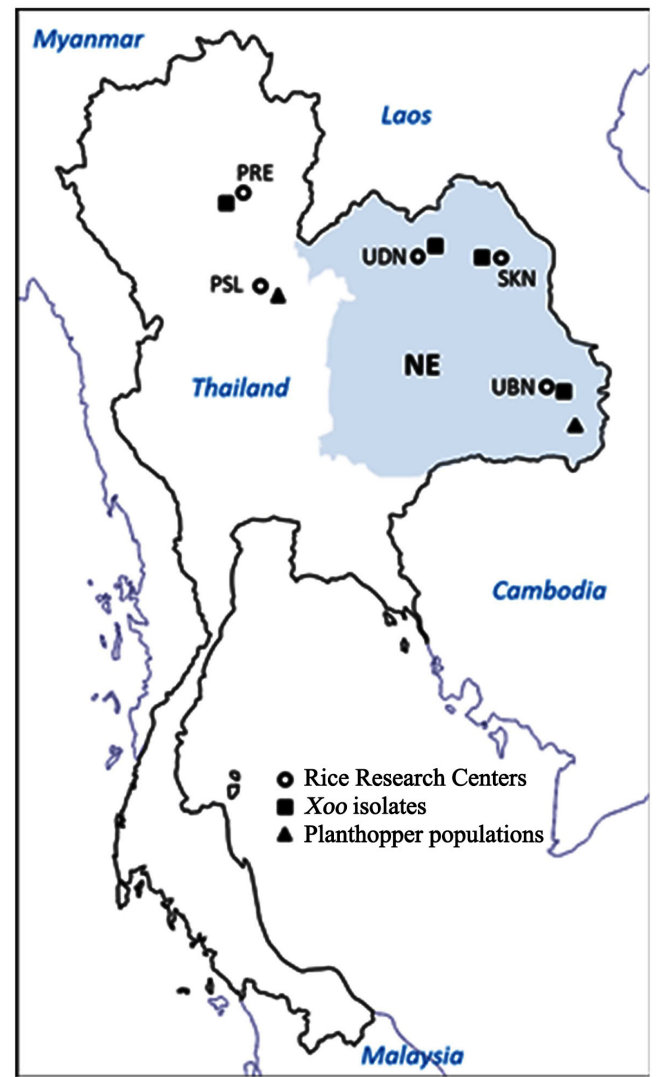

Figure 1. A map of trait evaluation sites $(\mathbf{O})$ and sample collection sites of the Xoo isolates ( $\mathbf{\Delta}$ ) and planthopper populations ( $\mathbf{\square})$ across north and northeast (NE) Thailand. Light gray areas represent the rainfed lowlands in the NE region.

\subsubsection{Bioassay for BB Resistance}

Xoo isolates collected from the Ubon Ratchathani (UBN), Sakon Nakhon (SKN), Udon Thani (UDN), and Phrae (PRE) provinces from 2016 to 2018 were used 
for BB resistance evaluation at the UBN-RRC, SKN-RRC, and RRE-RRC in the wet season (Figure 1). The isolate was grown in nutrient agar media for 72 hours at $28^{\circ} \mathrm{C}$. The bacterial cells were suspended in sterile water and adjusted to $\sim 108 \mathrm{CFU} / \mathrm{ml}$. Xoo isolates were assayed for a resistance reaction in RIL populations, susceptible (RD10, PSL2) and resistance (RD7, IRBB5, IRBB7) controls. $\mathrm{BB}$ inoculation was performed in a greenhouse using the leaf-clipping method [25]. Forty-five days after sowing, two to three fully expanded leaves of each RIL plant were inoculated. Resistance reactions were recorded based on the mean of the percent of the leaf area that appeared to be diseased [24] and the lesion length [26] of an individual plant 12 - 14 days after inoculation. The percentage of affected leaf area was used for SKN2016, UDN2016, and UBN2017, while leaf lesion length was used for UBN2016, SKN2018, and UDN2018 (Table 2). A damage score between 0 and 3 and a lesion length shorter than $5 \mathrm{~cm}$ were regarded as resistant to $\mathrm{BB}$.

\subsubsection{Bioassay for Planthopper Resistance}

RILs from the KD-IR71033 population were evaluated for WBPH resistance at the seedling stage and $\mathrm{BPH}$ resistance at the tilling stage. A WBPH population was collected from a rice field in the Phitsanulok (PSL) province in 2017 (Figure 1). The $\mathrm{WBPH}$ population was maintained on TN1 in a greenhouse at the Phitsanulok Rice Research Center (PSL-RRC). The seedbox screening test [27] was conducted to evaluate $\mathrm{WBPH}$ resistance in the test lines. Ten pre-germinated seeds of each test entry, susceptible (TN1) and resistance (PTB33) controls were sown in seedboxes $(60 \mathrm{~cm} \times 40 \mathrm{~cm} \times 10 \mathrm{~cm})$ containing well-puddled soil in the $12-\mathrm{cm}$ rows. Seven days after sowing, the seedlings were infested with second and third instar nymphs of WBPH at ten nymphs per seedling. When all seedlings of TN1 were almost dead, the SES scoring [24] was used to assign a resistance score to each genotype. A BPH population from a single colony was collected in the outbreak field from the UBN province in 2015 (Figure 1) and was grown on a susceptible variety of TN1 in a temperature-controlled rearing room $\left(25^{\circ} \mathrm{C} \pm 2^{\circ} \mathrm{C}\right)$ at the UBN-RRC. The screening method, which was modified from Jairin et al. [28], was conducted in a greenhouse at the UBN-RRC. The seeds of each RIL progeny, a susceptible cultivar (TN1), and a resistant cultivar (Rathu Heenati) were separately sown $(10 \times 20 \mathrm{~cm})$ in $7 \times 24 \mathrm{~m}^{2}$ seedling plots. Twenty days after sowing, the seedlings were infested with $3^{\text {rd }}-4^{\text {th }}$ instar nymphs of BPH at ten nymphs per seedling. Then, the insects fed, mated, laid eggs, and hatched freely. We evaluated the severity scores of the test lines according to SES [24] until TN1 and the susceptible recurrent parents died.

\subsection{Genotypic Data}

\subsubsection{DNA Extraction}

Samples included the parental and the two mapping populations, 286 RILs from KD-IR71033 and 288 RILs from KD-IR57514. Genomic DNA was extracted from the young leaves of each sample using the standard cetyltrimethylammo- 
nium bromide (CTAB) method [29] and quantified with a Nanodrop-1000 spectrophotometer (Thermo Fisher Scientific).

\subsubsection{SNP Genotyping}

A genotyping by sequencing (GBS) protocol using the ApeKI enzyme was applied to prepare the reduced representation libraries for sequencing. The forward adapters contained 9-bp unique barcodes in addition to the 21-bp Ion Forward adapter. The ApeKI restriction site was used to enable multiplex sequencing of the libraries. Genomic DNA digestion and adapter ligation were performed as described in Mascher et al. [30]. DNA fragments of 250 - $300 \mathrm{bp}$ were selected using E-Gel ${ }^{\mathrm{Tm}}$ SizeSelect ${ }^{\mathrm{Tm}}$ Agarose Gels (Invitrogen). The libraries were sequenced on the Ion S5 ${ }^{\text {Tm }}$ XL Sequencer (Thermo Fisher Scientific) with a loaded Ion $540^{\text {ma }}$ Chip according to the manufacturer's protocol. We multiplexed between 24 samples per run. All sequencing data generated in the present study were analyzed using Ion Torrent ${ }^{\text {th }}$ Suite Software Alignment Plugin V5.2.2 (Thermo Fisher Scientific) with the Nipponbare genome [31] as the alignment reference. The variants were called using the Torrent Variant Caller (Thermo Fisher Scientific). Filtered vcf files were imported into TASSEL 5 [32]. The default parameters of TASSEL were used to detect informative SNPs. SNPs were named according to their scaffold and base pair position within the Nipponbare genome.

\subsection{Linkage Map Construction}

The SNP markers with over 30\% missing data in the mapping population were excluded from analysis. Also excluded were SNPs that did not show polymorphisms in the parents but were polymorphic in the mapping populations. Segregation distortion of the individual markers was calculated using the Chi-square test on a 1:1 basis. Since segregation distortion has very little effect on marker order and map length [33], the SNPs showing segregation distortion with $\chi^{2}<10$ were included in the linkage analysis. The remaining SNP markers were used to construct genetic linkage maps in QTL IciMapping 4.1 [34] using the parameters set for the RIL population type. Marker groupings were manually anchored by their physical marker locations on the chromosomes. The filtered markers were then used to construct linkage maps with the MAP function in QTL IciMapping 4.1. The map distance (centimorgans, $\mathrm{cM}$ ) was converted by recombination fractions using the Kosambi function [35].

\subsection{QTL Analysis}

QTL maps of the BL, BB, WBPH, and $\mathrm{BPH}$ resistance phenotypes from the two RIL populations were generated using MapQTL 6.0 [36]. Chisquare goodness-of-fit was employed to fit the segregation pattern of the genotypic and phenotypic ratios. The multiple QTL model (MQM) mapping method was utilized to detect significant associations between phenotypic traits and marker da- 
ta sets. Significant LOD thresholds were determined using the permutation test at 1000 permutations per trait. The phenotypic variance explained (PVE) by a single QTL was estimated based on the population variance found within the progeny by a maximum likelihood estimation; those with a PVE greater than $15 \%$ were considered to be major QTLs.

\section{Results}

\subsection{Phenotypic Evaluation}

\subsubsection{BL Resistance}

Overall, 286 and 288 RILs from the KD-IR71033 and KD-IR57514 populations, respectively, were screened for $\mathrm{BL}$ resistance in the uniform $\mathrm{BL}$ nursery with natural infection at UBN-RRC, SKN-RRC, and PRE-RRC. IR71033 and IR57514 were resistant to BL, while KDML105 was highly susceptible in all of the nurseries. This indicated that IR71033 and IR57514 might be carrying broad field resistance against BL. The frequency distributions of the BL damage score for both RIL populations according to location and year are shown in Figure 2. The segregation of resistant and susceptible individuals in the KD-IR71033 population fitted a ratio of $1: 3\left(\mathrm{UBN} 2016 \chi^{2}=1.45, P=0.23\right.$; UBN2018 $\chi^{2}=3.53, P=0.06$; SKN2016 $\chi^{2}=3.08, P=0.08$; PRE2016 $\chi^{2}=1.29, P=0.26$ ), while in the KD-IR57514 population fitted a ratio of 3:1 (SKN2018 $\chi^{2}=3.17, P=0.07$; UBN2018 $\left.\chi^{2}=3.68, P=0.06\right)$.

\subsubsection{BB Resistance}

Two scoring methods were used to quantify BB resistance. In 2017 at UBN-RRC, an SES-based damage score was used to evaluate the resistance of the rice lines, while in 2016 and 2018 at UBN-RRC and SKN-RRC, the lesion length on the fully expanded leaves was used (Table 2). IR71033 and IR57514 were resistant to BB, while KDML105 was highly susceptible to all strains in all locations. The frequency distributions of $\mathrm{BB}$ damage scores and the lesion lengths for both RIL populations according to the location are presented in Figure 2. The segregation of resistant and susceptible individuals in the RIL populations showed a good fit to the expected ratio of 1:3 (KD-IR71033 population: UBN2016 $\chi^{2}=0.27, P=$ 0.60; UBN2017 $\chi^{2}=0.04, P=0.84$; SKN2018 $\chi^{2}=0.72, P=0.39$; UDN2016 $\chi^{2}=$ 2.14, $P=0.14$; UDN2018 $\chi^{2}=0.44, P=0.50$, KD-IR57514 population: UDN2018 $\chi^{2}=0.17, P=0.68$; SKN2018 $\left.\chi^{2}<0.01, P=0.97\right)$. The segregation pattern indicated that BB resistance derived from IR71033 and IR57514 was controlled by a single recessive gene.

\subsubsection{Planthopper Resistance}

The donor parent plant IR71033 displayed moderate resistance to WBPH and BPH, while KDML105 was completely susceptible to both insect pests. The level of resistance against BPH of RIL plants could not detected at seedling stage using standard seedbox screening test. Therefore, we decided to screen all RILs at the tillering stage for $\mathrm{BPH}$ resistance. The distribution of the damage scores for the 
progeny of KD-IR71033 was skewed towards susceptibility (Figure 2). The segregation of resistant to susceptible plants was in agreement to 1:3 segregation for $\mathrm{WBPH}$ at the seedling stage $\left(\chi^{2}=0.56, P=0.45\right)$ and $\mathrm{BPH}$ at the vegetative stage $\left(\chi^{2}<0.01, P=0.97\right)$. The segregation pattern indicated that planthopper resistance derived from the donor IR71033 was controlled by a single recessive gene.

\subsection{Construction of Linkage Maps}

\subsubsection{KD-IR71033 Mapping Population}

A total of 1897 polymorphic SNPs were used to construct the linkage map for KD-IR71033 and spanned a total genetic distance of $1899.7 \mathrm{cM}$ with linkage groups ranging from $69.9 \mathrm{cM}$ (chromosome 12) to $204.2 \mathrm{cM}$ (chromosome 11). The number of markers mapped to each chromosome varied from 108 (chromosome 9) to 223 (chromosome 1). An average of one SNP per cM region was detected across the genome with three large gaps on chromosomes 9,10 , and 11 (Figure 3).

\subsubsection{KD-IR57514 Mapping Population}

We used QTL IciMapping 4.1 to construct the linkage map for KD-IR57514 utilizing a total of 2237 polymorphic SNPs that spanned a total genetic distance of $2370.4 \mathrm{cM}$ on all of the 12 chromosomes. The number of markers mapped to each chromosome varied from 64 (chromosome 7) to 322 (chromosome 4). The genetic length of the smallest linkage group was $120.3 \mathrm{cM}$ on chromosome 9, and the largest was $311.3 \mathrm{cM}$ on chromosome 2 (Figure 3 ).
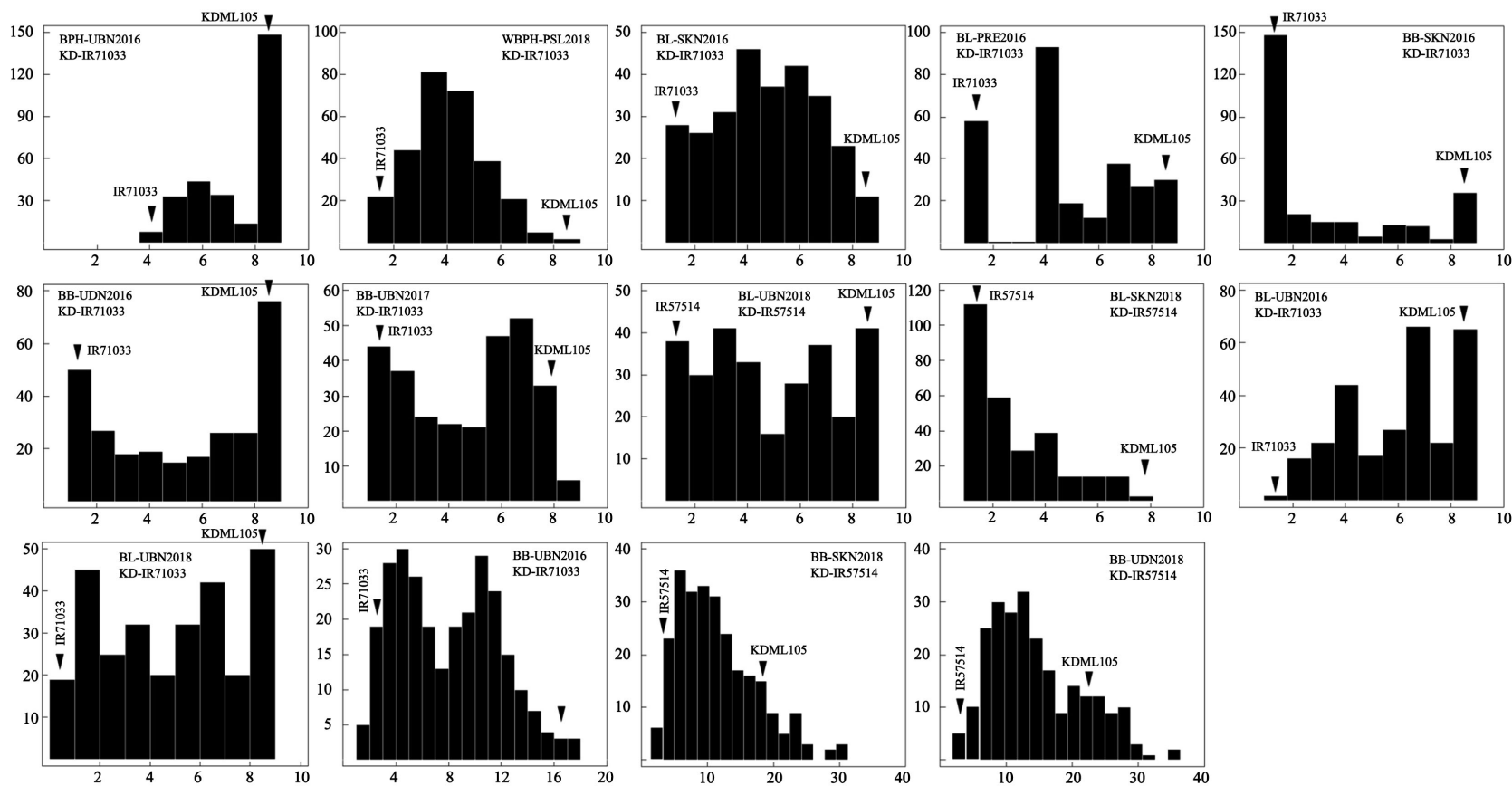

Figure 2. Frequency distribution of biotic stress resistance traits in the RIL population derived from the crosses of KDML105 $\times$ IR71033-121-15 and KDML105 × IR57514-PMI-5-B-1-2. 

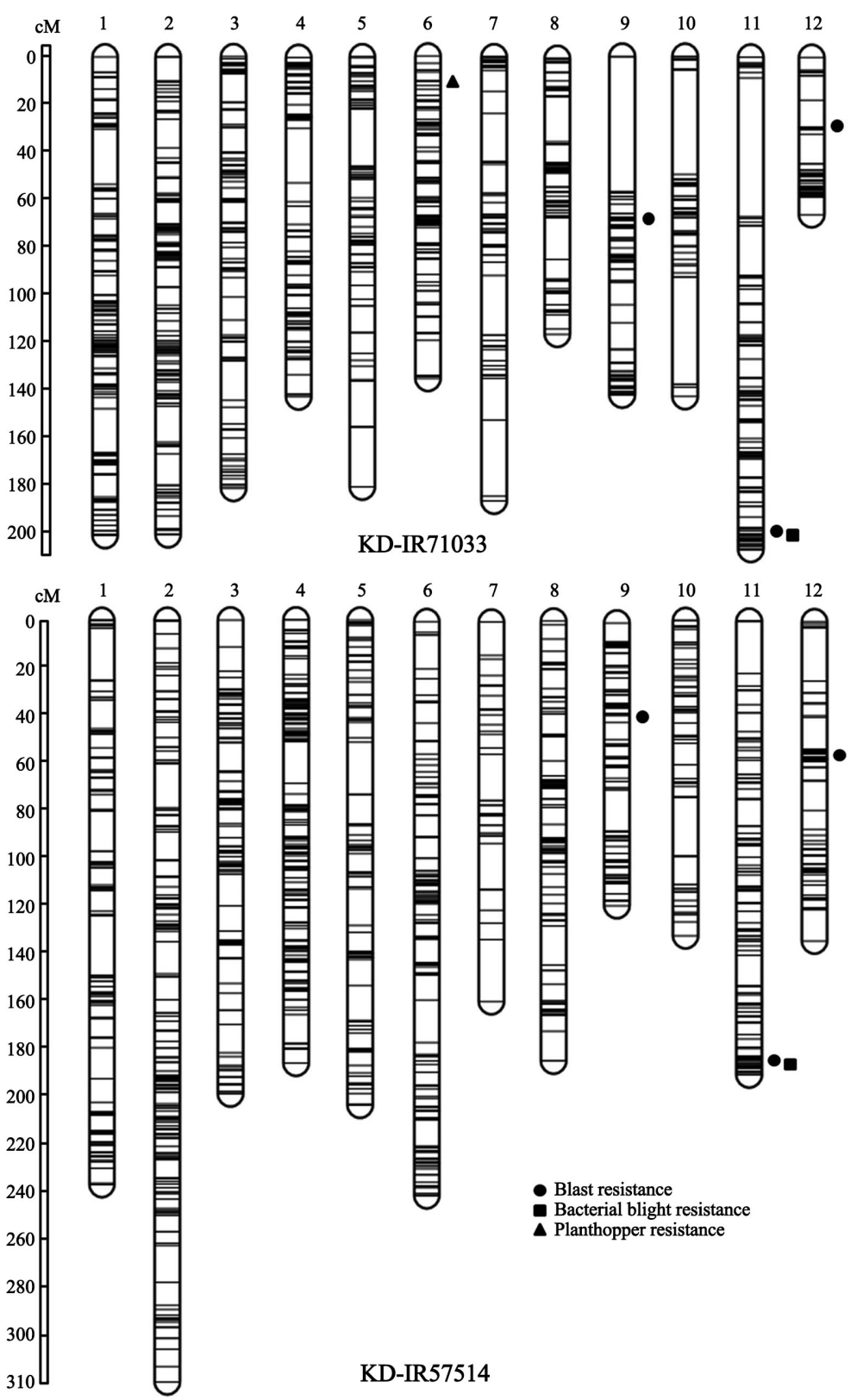

Figure 3. GBS-based genetic linkage maps of the two RIL populations from the crosses of KDML105 × IR71033-121-15 and KDML105 $\times$ IR57514-PMI-5-B-1-2. Chromosome numbers are indicated at the top of each map. Black triangles and square dots indicate the peak positions of the QTLs.

\subsection{QTL Analysis}

\subsubsection{QTL Mapping of BL Resistance Loci}

The linkage maps for KD-IR71033 and KD-IR57514 and the phenotypic trait evaluation data were used to map the QTLs associated with BL resistance. MQM mapping analysis detected QTLs for BL resistance in the same genomic regions for all of the three environments (UBN-RRC, SKN-RRC, and PRE-RRC) from 2016 to 2018. At UBN-RRC, QTL-mapping analysis revealed three QTLs associated with BL resistance on chromosomes 9, 11, and 12 of KD-IR71033 (in 2016 
and 2018) and KD-IR57514 (in 2018) mapping populations (Table 3). The percent of PVE for BL from these QTL ranged from $7.4 \%$ to $59.3 \%$ (Table 3). The major QTL from KD-IR71033 was located on chromosome 12 and flanked by SNP markers S12_8036165 and S12_10973592 in 2016 and S12_10254600 and S12_10973592 in 2018 with PVEs of $31.9 \%$ and 46.3\%, respectively. At SKN-RRC, the only major QTL was detected on chromosome 12 between S12_10060136 and S12_14061647 in 2016 for KD-IR71033 with a PVE of 19.7\%. In 2018, three QTLs were detected on chromosomes 9, 11, and 12 from KD-IR57514 at SKN-RRC with 6.1\%, 7.3\%, and 36.7\% PVE, respectively. From PRE-RRC in 2016, we identified three QTLs on chromosomes 9, 11, and 12 in KD-IR71033 with 19.3\%, 7.3\%, and 47.4\% PVE, respectively. Fewer QTLs were observed for BL at SKN-RRC than at other nurseries. All QTLs showed positive additive effect values, indicating that the alleles that confer resistance to $\mathrm{BL}$ are those from IR71033 and IR57514 (Table 3) and the major QTLs ( $q B L 1-12^{U B N 16}$, $\left.q B L 1-12^{U B N 18}, q B L 1-12^{S K N 16}, q B L 1-12^{P R E 16}, q B L 2-12^{U B N 18}, q B L 2-12^{S K N 18}\right)$ were mapped in the same Pi-ta region on chromosome 12.

\subsubsection{QTL Mapping of BB Resistance Locus}

The MQM mapping analysis detected major QTLs for $\mathrm{BB}$ resistance on chromosome 11 of both KD-IR71033 ( $q B B 1-11^{U D N 16}, q B B 1-11^{U B N 16}, q B B 1-11^{U B N 17}$, $\left.q B B 1-11^{S K N 16}\right)$ and KD-IR57514 ( $\left.q B B 1-11^{U D N 18}, q B B 1-11^{S K N 18}\right)$ with LODs ranging from 8.5 to 52.2 that explained $13.5 \%$ to $57.3 \%$ of the trait variations (Table 3). The QTL detected from KD-IR71033 was flanked with SNP markers S11_28162887 and S11_28977529 and from KD-IR57514 with S11_27446665 and S11_28704532. The QTLs detected from KD-IR71033 explained 43.9\%, 54.3\%, $40.7 \%$, and $13.5 \%$ of the PVE of $q B B 1-11^{U D N 16}, q B B 1-11^{U B N 16}, q B B 1-11^{U B N 17}$, and $q B B 1-11^{S K N 16}$, respectively. The QTL detected from KD-IR57514 in 2018 explained $28.2 \%$ and $41.7 \%$ of the PVE of $q B B 1-11^{U D N 18}$ and $q B B 1-11^{S K N 18}$, respectively (Table 3 ). The alleles that confer resistance to BB were from IR71033 and IR57514. The major QTL region was shared with the cluster region of Xa4 and Xa26.

\subsubsection{QTL Mapping of Planthopper Resistance Locus}

$\mathrm{WBPH}$ and $\mathrm{BPH}$ resistance evaluations were conducted in a greenhouse at PSL-RRC and UBN-RRC in 2018 and 2016, respectively. IR71033 was moderately resistant to the planthoppers, while IR57514 and KDML105 were completely susceptible. Therefore, only the KD-IR71033 mapping population was used to screen and map WBPH and BPH resistance loci. From QTL mapping, two QTLs ( $q W B P H 1-\sigma^{P S L 18}$ and $q B P H 1-6^{U B N 16}$ ) associated with WBPH and BPH resistance, respectively, were identified. The major QTL for WBPH and BPH were located between the SNP markers S6_841740 and S6_1394987 on the short arm of chromosome 6 and explained $19.7 \%$ and $61.8 \%$ of the phenotypic variation, respectively (Table 3 and Figure 3 ). The major QTL region was found to contain the published BPH-resistance gene Bph32. 
Table 3. Putative QTLs associated with $\mathrm{BL}, \mathrm{BB}, \mathrm{WBPH}$, and $\mathrm{BPH}$ resistance from the two RILs populations, KD-IR71033 and KD-IR57514.

\begin{tabular}{|c|c|c|c|c|c|c|c|}
\hline Trait & Location & $\mathrm{QTL}^{*}$ & $\mathrm{Chr}$ & Marker interval & LOD & PVE (\%) & Add \\
\hline \multicolumn{8}{|c|}{ KD-IR71033 } \\
\hline \multirow[t]{10}{*}{$\mathrm{BL}$} & $\mathrm{UBN} / 2016$ & $q B L 1-9^{U B N 16}$ & 9 & S9_8172820-S9_9643934 & 4.7 & 7.4 & 0.638 \\
\hline & & $q B L 1-11^{U B N 16}$ & 11 & S11_26529113-S11_27605483 & 4.6 & 7.4 & 0.634 \\
\hline & & $q B L 1-12^{U B N 16}$ & 12 & S12_8036165-S12_10973592 & 23.4 & 31.9 & 1.300 \\
\hline & $\mathrm{UBN} / 2018$ & $q B L 1-9^{U B N 18}$ & 9 & S9_9350745-S9_9643934 & 6.7 & 10.2 & 0.889 \\
\hline & & $q B L 1-11^{U B N 18}$ & 11 & S11_26529113-S11_27605483 & 8.1 & 12.2 & 0.957 \\
\hline & & $q B L 1-12^{U B N 18}$ & 12 & S12_10254600-S12_10973592 & 38.5 & 46.3 & 1.857 \\
\hline & SKN/2016 & $q B L 1-12^{5 K N 16}$ & 12 & S12_10060136-S12_14061647 & 13.4 & 19.7 & 1.029 \\
\hline & PRE/2016 & $q B L 1-9^{P R E 16}$ & 9 & S9_9586169-S9_9649453 & 13.1 & 19.3 & 1.211 \\
\hline & & $q B L 1-11^{P R E 16}$ & 11 & S11_26529113-S11_27605483 & 4.6 & 7.3 & 0.738 \\
\hline & & $q B L 1-12^{P R E 16}$ & 12 & S12_9375258-S12_13056239 & 39.2 & 47.4 & 1.860 \\
\hline \multirow[t]{4}{*}{ BB } & $\mathrm{UDN} / 2016$ & $q B B 1-11^{U D N 16}$ & 11 & S11_28162887-S11_28977529 & 31.1 & 40.7 & 1.886 \\
\hline & UBN/2016 & $q B B 1-11^{U B N 16}$ & 11 & S11_28162887-S11_28977529 & 34.6 & 43.9 & 2.610 \\
\hline & $\mathrm{UBN} / 2017$ & $q B B 1-11^{U B N 17}$ & 11 & S11_28162887-S11_28977529 & 34.6 & 43.9 & 1.716 \\
\hline & SKN/2016 & $q B B 1-11^{S K N 16}$ & 11 & 11_28162887-S11_28977529 & 8.5 & 13.5 & 1.093 \\
\hline $\mathrm{BPH}$ & $\mathrm{UBN} / 2016$ & $q B P H 1-6^{U B N 16}$ & 6 & S6_1045301-S6_1394987 & 58.1 & 61.4 & 1.269 \\
\hline WBPH & PSL/2018 & $q W B P H 1-\sigma^{P S L 18}$ & 6 & S6_841740-S6_1394987 & 13.7 & 19.7 & 0.663 \\
\hline \multicolumn{8}{|c|}{ KD-IR57514 } \\
\hline \multirow[t]{6}{*}{ BL } & $\mathrm{UBN} / 2018$ & $q B L 2-9^{U B N 18}$ & 9 & S9_8172820-S9_9911937 & 6.5 & 10.0 & 0.907 \\
\hline & & $q B L 2-11^{U B N 18}$ & 11 & S11_26570630-S11_27584310 & 8.3 & 12.5 & 0.965 \\
\hline & & $q B L 2-12^{U B N 18}$ & 12 & S12_10254695-S12_12601441 & 55.4 & 59.3 & 2.084 \\
\hline & SKN/2018 & $q B L 2-9^{S K N 18}$ & 9 & S9_8172820-S9_9911937 & 6.1 & 9.4 & 0.616 \\
\hline & & $q B L 2-11^{S K N 18}$ & 11 & S11_25633354-S11_27030149 & 4.7 & 7.3 & 0.511 \\
\hline & & $q B L 2-12^{S K N 18}$ & 12 & S12_9375258-S12_10419846 & 28.2 & 36.7 & 1.146 \\
\hline \multirow[t]{2}{*}{ BB } & UDN/2018 & $q B B 2-11^{U D N 18}$ & 11 & S11_27446665-S11_28704532 & 20.4 & 28.2 & 2.813 \\
\hline & SKN/2018 & $q B B 2-11^{S K N 18}$ & 11 & S11_27446665-S11_28704532 & 32.9 & 41.7 & 3.263 \\
\hline
\end{tabular}

PVE: percent phenotypic variation; Add: additive effect; * The numbers "1" and " 2 " before the hyphen indicates the RIL populations of KD-IR71033 and KD-IR57514, respectively.

\subsubsection{Number of Predicted Genes Anchored within Targeted QTLS}

For BL resistance, the QTLs $q B L 1-9$ and $q B L 2-9$ on chromosome 9 from IR71033 and IR57514 were detected in the regions between SNP markers S9_8172820 and S9_9911937. There are 93 annotated genes within this region in the Nipponbare genome including three BL resistance genes ( $P i 3, P i 5$, and Pi15). The $q B L 1-11$ and $q B L 2-11$ regions between the S11_25260851 and S11_27584310 markers on chromosome 11 contain 85 predicted genes, which include the Pik and Pi44 genes. The major QTLs ( $q B L 1-12^{U B N 16}, q B L 1-12^{U B N 1}$, 
$q B L 1-12^{S K N 16}, q B L 1-12^{P R E 16}, q B L 2-12^{U B N 18}, q B L 2-12^{S K N 18}$ ) were in the Pi-ta region. The 718.9-kb QTL region contains 44 predicted genes.

For $\mathrm{BB}$ resistance, the $q B B 1-11$ and $q B B 2-11$ regions contain 85 predicted genes including the $X a 3 / X a 26, X a 4, X a 40$, and $x a 44$ genes. The QTLs from IR71033 $q W B P H 1-6$ and $q B P H 1-6$ associated with WBPH and BPH resistance, respectively, were detected in the Bph32 genomic region between S6_1045301 and S6_1394987 on chromosome 6. This 349.7-kb region of the Nipponbare genome contains 39 predicted genes.

\section{Discussion}

The $\mathrm{BL}, \mathrm{BB}, \mathrm{WBPH}$, and $\mathrm{BPH}$ pathogens are the most devastating for the rice plants in rainfed lowland of NE Thailand [1] [2]. KDML105 and its induced-mutation varieties (RD15 and RD6) have been wildly grown in this rainfed lowland area, which occupies approximately 5.7 million hectares [1]. All varieties have the same genetic background and are highly susceptible to $\mathrm{BL}, \mathrm{BB}$, $\mathrm{WBPH}$, and $\mathrm{BPH}$ due to their lack of resistance genes. The most efficient strategy to manage harmful pests is enhancing the host plant's resistance to these pathogenic organisms [37]. Therefore, breeding toward durable resistance against a broad spectrum of these pests is the most practical approach for preventing crop loss [38]. A large number of genes/QTLs for $\mathrm{BL}, \mathrm{BB}, \mathrm{WBPH}$, and $\mathrm{BPH}$ resistance have been identified and used in rice breeding programs over the past two decades [8] [13] [39] [40]. Recently, GBS has greatly improved the discovery of QTLs. GBS is a rapid and cost-effective means for identifying SNPs to generate high-density linkage maps [41]. In the present study, we successfully employed the GBS method to detect SNPs linked to novel pest resistance QTLs in the RIL populations. Most of the major resistance QTLs detected in this study is co-located in the same genomic regions of resistance genes identified in previous studies [7] [12] [17] [20] [42] [43] [44].

The introgression lines derived from $O$. minuta have served as a source for biotic stress resistance genes [17] [18] [19] [20]. Several QTLs for BL and BPH resistance have been identified from IR71033. Five QTLs on chromosomes 6, 7, 9 and 11 for BL resistance were identified in a previous study [20]. The major QTLs on chromosomes 9 and 11 were located in the Pi2/9 [45] and Pik/Pikm [46] regions, respectively. Minor QTLs for BL resistance were detected in the present study in the same regions on chromosomes 9 and 11 from all three environments investigated. Furthermore, we detected a major QTL for BL resistance on chromosome 12 that explained $19.7 \%-47.4 \%$ of the phenotypic variation across all of the BL nurseries. The major QTLs are co-located in the Pi-ta region located in a linkage block near the centromere, which contains three recently-described genes, Pi-ta, Pi-ta2, and Ptr [9] [47]. The Pi-ta resistance complex likely contributes to the resistance of IR71033, though this requires further study.

IR71033 showed moderate to high resistance against planthoppers [17] [18] 
[19]. We mapped the QTLs associated with WBPH and BPH resistance in the same genomic region on the short arm of chromosome 6. A major QTL for BPH resistance in IR71033 has been reported on chromosome 6 [18]. Later, two major QTLs for BPH resistance in IR71033 were discovered on chromosomes 4 and 12 [19]. Furthermore, a moderately resistant QTL from IR71033-62-24, another introgression line derived from $O$. minuta, was mapped onto chromosome 6 [17]. The present study confirms the location of a major QTL for BPH resistance in the Bph32 region on chromosome 6 [48]. It was reassuring to find that different QTLs from the same donor can be detected by multiple, independent studies [42] [49]. The detection of different QTLs might be influenced by factors including the nature of the phenotypic tests and environments, a complex of pathogen or insect biotypes, genetic background, population types, and size or number of markers [50] [51].

IR57514 is widely and well adapted in the low-yielding environment of the rainfed lowland throughout Asia [22]. It has been extensively used in experiments and breeding programs for abiotic tolerance in these areas [52] [53] [54]. This rice line was identified as a submergence-tolerant variety that carries the Sub1 locus [52]. QTLs for submergence and drought tolerance-related traits have been detected in IR57514 (Bureerat et al., unpublished data). In addition to abiotic traits, IR57514 also carries resistance genes against BL and BB. QTLs for BL resistance have been detected in the same chromosomal locations of IR71033. In the present study, a novel gene conferring resistance against BB from IR57514 and IR71033 was identified simultaneously in the same genomic regions from three environments and across three years of evaluation experiments. Two QTLs ( $q B B 1-11, q B B 2-11)$ were detected in the distal region of chromosome 11 in a previous study [12]. Several BB resistance genes were reported in this region (approximately $300 \mathrm{Mb}$ ), including Xa4, xa44, Xa40, and Xa3/Xa26 [12] [55] [56] [57].

Our results suggest that IR71033 and IR57514 could potentially provide multiple important genes for biotic stress resistance including against $\mathrm{BL}, \mathrm{BB}$, $\mathrm{WBPH}$, and BPH in multiple environments. Previous studies suggested that multiple genes control durable field resistance to rice pathogens [58] [59]. Therefore, the use of field resistance is one of the most promising measures for pest control [60] [61]. QTLs for field resistance identified in this study will be useful for breeding programs aimed to protect rice from diseases and insect pests toward ensuring stable rice production through the MAS. Pyramiding these QTLs may address the problem of resistance breakdown leading to the development of multiple broad-spectrum, durable biotic stress resistant cultivars, particularly in the rainfed lowland areas.

\section{Conclusion}

In conclusion, in this study, major QTLs for $\mathrm{BL}, \mathrm{BB}, \mathrm{WBPH}$, and $\mathrm{BPH}$ resistance were detected from two RIL populations, KD-IR71033 and KD-IR57514, from 
distinct environments at multiple time points. Major BB and BL resistance QTLs occupy the genomic regions on chromosomes 11 and 12 respectively, while WBPH and BPH resistance QTLs are identified on chromosome 6. Almost all of the QTLs detected in this study were co-located in the same regions as previously reported resistance genes: Pi5 (chromosome 9), Pi44 (chromosome 11), Pi-ta (chromosome 12), xa44 (chromosome 11), and Bph32 (chromosome 6). The RILs and markers flanking the target regions should be valuable for improving durable $\mathrm{BL}, \mathrm{BB}, \mathrm{WBPH}$, and $\mathrm{BPH}$ resistance in rice through marker-assisted breeding. Taken together, these efforts should reduce the impact of biotic pathogens on local farmers worldwide.

\section{Acknowledgements}

This research was supported by a research grant from the Rice Department, Thailand (Project No. 705381). We are thankful to the International Rice Research Institute for providing seeds for the elite rice lines used in this study. We thank our colleagues at the UBN-RRC, SKN-RRC, PRE-RRC, PSL-RRC, and UDN-RRC for their assistance with both laboratory and field work.

\section{Conflicts of Interest}

The authors declare no conflicts of interest regarding the publication of this paper.

\section{References}

[1] Jairin, J., Kotchasatit, U., Saleeto, S., Jearakongman, S., Srivillai, K., Chamarerk, V., Kotcharerk, J., Pattawatang, P., Korinsak, S., Wongsaprom, C. and Toojinda, T. (2017) Application of Marker-Assisted Breeding to Improve Biotic Stress Resistance for Rainfed Lowland Rice in Northeastern Thailand. SABRAO Journal of Breeding and Genetics, 49, 168-178.

[2] Vanavichit, A., Kamolsukyeunyong, W., Siangliw, M., Siangliw, J.L., Traprab, S., Ruengphayak, S., Chaichoompu, E., Saensuk, C., Phuvanartnarubal, E., Toojinda, T. and Tragoonrung, S. (2018) Thai Hom Mali Rice: Origin and Breeding for Subsistence Rainfed Lowland Rice System. Rice, 11, 20.

https://doi.org/10.1186/s12284-018-0212-7

[3] Sansen, K., Wongboon, W., Jairin, J. and Kato, Y. (2019) Farmer-Participatory Evaluation of Mechanized Dry Direct-Seeding Technology for Rice in Northeastern Thailand. Plant Production Science, 22, 46-53. https://doi.org/10.1080/1343943X.2018.1557530

[4] Jairin, J. (2018) Genetic Improvement of Thai Aromatic Rice for Rainfed Lowlands through Molecular Breeding. In: Proceedings of the 4th International Plant Breeding Conference, Genetics Society of Malaysia, Selangor.

[5] Bonman, J.M., Khush, G.S. and Nelson, R.J. (1992) Breeding Rice for Resistance to Pests. Annual Review of Phytopathology, 30, 507-528. https://doi.org/10.1146/annurev.py.30.090192.002451

[6] Zhang, Q. (2007) Strategies for Developing Green Super Rice. Proceedings of the National Academy of Sciences of the United States of America, 104, 42. 
https://doi.org/10.1073/pnas.0708013104

[7] Ballini, E., Morel, J.B., Droc, G., Price, A., Courtois, B., Notteghem, J.L. and Tharreau, D. (2008) A Genome-Wide Meta-Analysis of Rice Blast Resistance Genes and Quantitative Trait Loci Provides New Insights into Partial and Complete Resistance. Molecular Plant-Microbe Interactions, 21, 859-868.

https://doi.org/10.1094/MPMI-21-7-0859

[8] Debnath, A., Bhutia, K.L. and Sumpi, H. (2018) Mapping and Deployment of Blast Resistance Gene in Rice-A Work in Progress. International Journal of Current Microbiology and Applied Sciences, 7, 2073-2094. https://doi.org/10.20546/ijcmas.2018.705.243

[9] Zhao, H., Wang, X., Jia, Y., Minkenberg, B., Wheatley, M., Fan, J., Jia, M.H., Famoso, A., Edwards, J.D., Wamishe, Y., Valent, B., Wang, G.L. and Yang, Y. (2018) The Rice Blast Resistance Gene Ptr Encodes an Atypical Protein Required for Broad-Spectrum Disease Resistance. Nature Communications, 9, 2039.

https://doi.org/10.1038/s41467-018-04369-4

[10] Busungu, C., Taura, S., Sakagami, J. and Ichitani, K. (2016) Identification and Linkage Analysis of a New Rice Bacterial Blight Resistance Gene from XM14, a Mutant Line from IR24. Breeding Science, 66, 636-645. https://doi.org/10.1270/jsbbs.16062

[11] Dilla-Ermita, C.J., Tandayu, E., Juanillas, V.M., Detras, J., Lozada, D.N., Dwiyanti, M.S., Cruz, C.V., Mbanjo, E.G.N, Ardales, E., Diaz, M.G., Mendioro, M., Thomson, M.J. and Kretzschmar, T. (2017) Genome-Wide Association Analysis Tracks Bacterial Leaf Blight Resistance Loci in Rice Diverse Germplasm. Rice, 10, 8. https://doi.org/10.1186/s12284-017-0147-4

[12] Kim, S.M. (2018) Identification of Novel Recessive Gene xa44(t) Conferring Resistance to Bacterial Blight Races in Rice by QTL Linkage Analysis Using an SNP Chip. Theoretical and Applied Genetics, 12, 2733-2743. https://doi.org/10.1007/s00122-018-3187-2

[13] Fujita, D., Kohli, A. and Horgan, F.G. (2013) Rice Resistance to Planthoppers and Leafhoppers. Critical Reviews in Plant Sciences, 32, 3. https://doi.org/10.1080/07352689.2012.735986

[14] Jairin, J., Teangdeerith, S., Leelagud, P., Kothcharerk, J., Sansen, K., Yi, M., Vanavichit, A. and Toojinda, T. (2009) Development of Rice Introgression Lines with Brown Planthopper Resistance and KDML105 Grain Quality Characteristics through Marker-Assisted Selection. Field Crops Research, 110, 263-271. https://doi.org/10.1016/j.fcr.2008.09.009

[15] Kumar, K.K., Kokiladevi, E., Arul, L., Varanavasiappan, S. and Sudhakar, D. (2018) Engineering Disease Resistance in Rice. In: Gosal, S. and Wani, S., Eds., Biotechnologies of Crop Improvement, Volume 2, Springer, Cham, 183-206. https://doi.org/10.1007/978-3-319-90650-8_8

[16] Horgan, F.G. (2018) Integrating Gene Deployment and Crop Management for Improved Rice Resistance to Asian Planthoppers. Crop Protection, 110, 21-33. https://doi.org/10.1016/j.cropro.2018.03.013

[17] Harini, A.S., Lakshmi, S.S., Kumar, S.S., Sivaramakrishnan, S. and Kadirvel, P. (2010) Validation and Fine-Mapping of Genetic Locus Associated with Resistance to Brown Plant Hopper [Nilaparvata lugens (Stal.)] in Rice (Oryza sativa L.). Asian Journal of Bio Science, 5, 32-37.

[18] Jairin, J., Teangdeerith, S., Leelagud, P., Phengrat, P., Vanavichit, A. and Toojinda T. (2007) Detection of Brown Planthopper Resistance Genes from Different Rice Mapping Populations in the Same Genomic Location. Science Asia, 33, 347-352. 
https://doi.org/10.2306/scienceasia1513-1874.2007.33.347

[19] Rahman, M.L., Jiang, W., Chu, S.H., Qiao, Y., Ham, T.H., Woo, M.O., Lee, J., Khanam, M.S., Chin, J.H., Jeung, J.U., Brar, D.S., Jena, K.K. and Koh, H.J. (2009) High Resolution Mapping of Two Rice Brown Planthopper Resistance Genes, Bph20(t) and Bph21(t), Originating from Oryza minuta. Theoretical and Applied Genetics, 119, 1237-1246. https://doi.org/10.1007/s00122-009-1125-z

[20] Rahman, L., Khanami, S., Roh, J.H. and Koh, H.J. (2011) Mapping of QTLs Involved in Resistance to Rice Blast (Magnaporthe grisea) Using Oryza minuta Introgression Lines. Czech Journal of Genetics and Plant Breeding, 47, 85-94. https://doi.org/10.17221/19/2011-CJGPB

[21] Kotchasatit, A. (2015) IR57514-PMI-5-B-1-2: Elite Line for Climate Change. In: Proceedings of the Rice and Temperate Cereal Crops Annual Conference, NE Rice Research Center Cluster, Ubon Ratchathani, Thailand, 60-74.

[22] Wade, L.J., McLaren, C.G., Quintana, L., Harnpichitvitaya, D., Rajatasereekul, S., Sarawgi, A.K., Kumar, A., Ahmed, H.U., Sarwoto, Singh, A.K., Rodriguez, R., Siopongco, J. and Sakarung, S. (1999) Genotype by Environment Interactions across Diverse Rainfed Lowland Rice Environments. Field Crops Research, 64, 35-50. https://doi.org/10.1016/S0378-4290(99)00049-0

[23] Elshire, R.J., Glaubitz, J.C., Sun, Q., Poland, J.A., Kawamoto, K., Buckler, E.S. and Mitchell, S.E. (2011) A Robust, Simple Genotyping-by-Sequencing (GBS) Approach for High Diversity Species. PLoS ONE, 6, e19379.

https://doi.org/10.1371/journal.pone.0019379

[24] IRRI (2013) Standard Evaluation System for Rice. International Rice Research Institute, Manila.

[25] Kauffman, H.E., Reddy, A.P.K., Hsieh, S.P.Y. and Merca, S.D. (1973) An Improved Technique for Evaluating Resistance of Rice Varieties to Xanthomonas oryzae. Plant Disease Report, 57, 737-741.

[26] Amante-Bordeos, A., Sitch, L.A., Nelson, R., Dalmacio, R.D., Oliva, N.P., Aswidinnoor, H. and Leung, H. (1992) Transfer of Bacterial Blight and Blast Resistance from the Tetraploid Wild Rice Oryza minuta to Cultivated Rice, Oryza sativa. Theoretical and Applied Genetics, 84, 345-354. https://doi.org/10.1007/BF00229493

[27] Heinrichs, E.A., Medrano, F.G. and Rapusas, H.R. (1985) Genetic Evaluation for Insect Resistance in Rice. IRRI, Los Baños.

[28] Jairin, J., Teangdeerith, S., Leelagud, P., Phengrat, K., Vanavichit, A. and Toojinda, T. (2007) Physical Mapping of Bph3, a Brown Planthopper Resistance Locus in Rice. Maejo International Journal of Science and Technology, 1, 166-177.

[29] Doyle, J.J. and Doyle, J.L. (1987) A Rapid DNA Isolation Procedure for Small Quantities of Fresh Leaf Tissue. Phytochemical Bulletin, 19, 11-15.

[30] Mascher, M., Wu, S., Amand, P.S., Stein, N. and Poland, J. (2013) Application of Genotyping-by-Sequencing on Semiconductor Sequencing Platforms: A Comparison of Genetic and Reference-Based Marker Ordering in Barley. PLOS ONE, 8, e76925. https://doi.org/10.1371/journal.pone.0076925

[31] Kawahara, Y., de la Bastide, M., Hamilton, J.P., Kanamori, H., McCombie, W.R., Ouyang, S., Schwartz, D.C., Tanaka, T., Wu, J., Zhou, S., Childs, K.L., Davidson, R.M., Lin, H., Quesada-Ocampo, L., Vaillancourt, B., Sakai, H., Lee, S.S., Kim, J., Numa, H., Itoh, T., Buell, C.R. and Matsumoto, T. (2013) Improvement of the Oryza sativa Nipponbare Reference Genome Using Next Generation Sequence and Optical Map Data. Rice, 6, 4. https://doi.org/10.1186/1939-8433-6-4

[32] Bradbury, P.J., Zhang, Z., Kroon, D.E., Casstevens, T.M., Ramdoss, Y. and Buckler, 
E.S. (2007) TASSEL: Software for Association Mapping of Complex Traits in Diverse Samples. Bioinformatics, 23, 2633-2635.

[33] Hackett, C.A. and Broadfoot, L.B. (2003) Effects of Genotyping Errors, Missing Values and Segregation Distortion in Molecular Marker Data on the Construction of Linkage Maps. Heredity, 90, 33-38. https://doi.org/10.1038/sj.hdy.6800173

[34] Meng, L., Li, H.H., Zhang, L.Y. and Wang, J.K. (2015) QTL IciMapping: Integrated Software for Genetic Linkage Map Construction and Quantitative Trait Locus Mapping in Biparental Populations. The Crop Journal, 3, 269-283. https://doi.org/10.1016/j.cj.2015.01.001

[35] Kosambi, D.D. (1944) The Estimation of Map Distance from Recombination Values. Annals of Eugenics, 12, 172-175. https://doi.org/10.1111/j.1469-1809.1943.tb02321.x

[36] Van Ooijen, J.W. (2004) MapQTL 5, Software for the Mapping of Quantitative Trait Loci in Experimental Populations. Kyazma B.V., Wageningen.

[37] Kourelis, J. and van der Hoorn, R.A.L. (2018) Defended to the Nines: 25 Years of Resistance Gene Cloning Identifies Nine Mechanisms for R Protein Function. Plant Cell, 30, 285-299. https://doi.org/10.1105/tpc.17.00579

[38] Mekonnen, T., Haileselassie, T. and Tesfaye, K. (2017) Identification, Mapping and Pyramiding of Genes/Quantitative Trait Loci (QTLs) for Durable Resistance of Crops to Biotic Stresses. Journal of Plant Pathology and Microbiology, 8, 412. https://doi.org/10.4172/2157-7471.1000412

[39] Li, X., Zhai, H., Wan, J., Ma, L., Zhuang, J., Liu, G. and Yang, C. (2004) Mapping of a New Gene Wbph6(t) Resistant to the Whitebacked Planthopper, Sogatella furcifera, in Rice. Rice Science, 11, 86-90.

[40] Tan, G.X., Weng, Q.M., Ren, X., Huang, Z., Zhu, L.L. and He, G.C. (2004) Two Whitebacked Planthopper Resistance Genes in Rice Share the Same Loci with Those for Brown Planthopper Resistance. Heredity, 92, 212-217. https://doi.org/10.1038/sj.hdy.6800398

[41] He, J., Zhao, X., Laroche, A., Lu, Z.X., Liu, H.K. and Li, Z. (2014) Genotyping-by-Sequencing (GBS), an Ultimate Marker-Assisted Selection (MAS) Tool to Accelerate Plant Breeding. Frontiers in Plant Science, 5, 484. https://doi.org/10.3389/fpls.2014.00484

[42] Jairin, J., Phengrat, K., Teangdeerith, S., Vanavichit, A. and Toojinda, T. (2007) Mapping of a Broad-Spectrum Brown Planthopper Resistance Gene, Bph3, on Rice Chromosome 6. Molecular Breeding, 19, 35-44. https://doi.org/10.1007/s11032-006-9040-3

[43] Kang, K., Wang, Y., Peng, S., Zhang, Y., Xiao, Y., Wang, D., Qu, S., Li, Z., Yan, S., Wang, Z., Liu, W., Ning, Y., Korniliev, P., Leung, H., Mezey, J., McCouch, S.R. and Wang, G.L. (2016) Dissection of the Genetic Architecture of Rice Resistance to the Blast Fungus Magnaporthe oryzae. Molecular Plant Pathology, 17, 959-972. https://doi.org/10.1111/mpp.12340

[44] Pan, Q.H., Hu, Z.D., Takatoshi, T. and Wang, L. (2003) Fine Mapping of the Rice Blast Resistance Gene Pi15, Linked to Pii, on Rice Chromosome 9. Acta Botanica Sinica, 45, 871-877.

[45] Jiang, N., Li, Z., Wu, J., Wang, Y., Wu, L., Wang, S., Wang, D., Wen, T., Liang, Y., Sun, P., Liu, J., Dai, L., Wang, Z., Wang, C., Luo, M., Liu, X. and Wang, G.L. (2012) Molecular Mapping of the Pi2/9 Allelic Gene Pi2-2 Conferring Broad-Spectrum Resistance to Magnaporthe oryzae in the Rice Cultivar Jefferson. Rice, 5, 29. https://doi.org/10.1186/1939-8433-5-29 
[46] Ashikawa, I., Hayashi, N., Abe, F., Wu, J. and Matsumoto, T. (2012) Characterization of the Rice Blast Resistance Gene Pik Cloned from Kanto51. Molecular Breeding, 30, 485-494. https://doi.org/10.1007/s11032-011-9638-y

[47] Jia, Y. and Martin, R. (2008) Identification of a New Locus, Ptr(t), Required for Rice Blast Resistance Gene Pi-ta-Mediated Resistance. Molecular Plant-Microbe Interactions, 21, 396-403. https://doi.org/10.1094/MPMI-21-4-0396

[48] Ren, J., Gao, F., Wu, X., Lu, X., Zeng, L., Lv, J., Su, X., Luo, H. and Ren, G. (2016) Bph32, a Novel Gene Encoding an Unknown SCR Domain-Containing Protein, Confers Resistance against the Brown Planthopper in Rice. Scientific Reports, 6, Article No. 37645. https://doi.org/10.1038/srep37645

[49] Sun, L., Su, C., Wang, C., Zhai, H. and Wan, J. (2005) Mapping of a Major Resistance Gene to the Brown Planthopper in the Rice Cultivar Rathu Heenati. Breeding Science, 55, 391-396. https://doi.org/10.1270/jsbbs.55.391

[50] He, P., Li, J.Z., Zheng, X.W., Shen, L.S., Lu, C.F., Chen, Y. and Zhu, L.H. (2001) Comparison of Molecular Linkage Maps and Agronomic Trait Loci between DH and RIL Populations Derived from the Same Rice Cross. Crop Science, 41, 1240-1246. https://doi.org/10.2135/cropsci2001.4141240x

[51] Lu, C., Shen, L., Tan, Z., Xu, Y., He, P., Chen, Y. and Zhu, L. (1996) Comparative Mapping of QTLs for Agronomic Traits of Rice across Environments Using a Doubled Haploid Population. Theoretical and Applied Genetics, 93, 1211-1217. https://doi.org/10.1007/BF00223452

[52] Jantaboon, J., Siangliw, M., Im-mark, S., Jamboonsri, W., Vanavichit, A. and Toojinda, T. (2011) Ideotype Breeding for Submergence Tolerance and Cooking Quality by Marker-Assisted Selection in Rice. Field Crops Research, 123, 206-213. https://doi.org/10.1016/j.fcr.2011.05.001

[53] Jongdee, B., Pantuwan, G., Fukai, S. and Fischer, K. (2006) Improving Drought Tolerance in Rainfed Lowland Rice: An Example from Thailand. Agricultural Water Management, 80, 225-240. https://doi.org/10.1016/j.agwat.2005.07.015

[54] Ouk, M., Basnayake, J., Tsubo, M., Fukai, S., Fischer, K.S., Cooper, M. and Nesbitt, H. (2006) Use of Drought Response Index for Identification of Drought Tolerant Genotypes in Rainfed Lowland Rice. Field Crops Research, 99, 48-58. https://doi.org/10.1016/j.fcr.2006.03.003

[55] Kim, S.M., Suh, J.P., Qin, Y., Noh, T.H., Reinke, R.F. and Jena, K.K. (2015) Identification and Fine-Mapping of a New Resistance Gene, Xa40, Conferring Resistance to Bacterial Blight Races in Rice (Oryza sativa L.). Theoretical and Applied Genetics, 128, 1933-1943. https://doi.org/10.1007/s00122-015-2557-2

[56] Li, H.J., Li, X.H., Xiao, J.H., Wing, R.A. and Wang, S.P. (2012) Ortholog Alleles at Xa3/Xa26 Locus Confer Conserved Race-Specific Resistance against Xanthomonas oryzae in Rice. Molecular Plant, 5, 281-290. https://doi.org/10.1093/mp/ssr079

[57] Sun, X., Yang, Z., Wang, S. and Zhang, Q. (2003) Identification of a 47-kb DNA Fragment Containing Xa4, a Locus for Bacterial Blight Resistance in Rice. Theoretical and Applied Genetics, 106, 683-687. https://doi.org/10.1007/s00122-002-1117-8

[58] Fukuoka, S., Saka, N., Mizukami, Y., Koga, H., Yamanouchi, U., Yoshioka, Y., Hayashi, N., Ebana, K., Mizobuchi, R. and Yano, M. (2015) Gene Pyramiding Enhances Durable Blast Disease Resistance in Rice. Scientific Reports, 5, Article No. 7773. https://doi.org/10.1038/srep07773

[59] Li, Z.K., Luo, L.J., Mei, H.W., Paterson, A.H., Zhao, X.H., Zhong, D.B., Wang, Y.P., Yu, X.Q., Zhu, L., Tabien, R., Stansel, J.W. and Ying, C.S. (1999) A “Defeated” Rice Resistance Gene Acts as a QTL against a Virulent Strain of Xanthomonas oryzae pv. 
oryzae. Molecular and General Genetics, 261, 58-63.

https://doi.org/10.1007/s004380050941

[60] Miyamoto, M., Yano, M. and Hirasawa, H. (2001) Mapping of Quantitative Trait Loci Conferring Blast Field Resistance in the Japanese Upland Rice Variety Kahei. Breeding Science, 51, 257-261. https://doi.org/10.1270/jsbbs.51.257

[61] Sato, H., Takeuchi, Y., Hirabayashi, H., Nemoto, H., Hirayama, M., Kato, H., Imbe, T. and Ando, I. (2006) Mapping QTLs for Field Resistance to Rice Blast in the Japanese Upland Rice Variety Norin 12. Breeding Science, 56, 415-418.

https://doi.org/10.1270/jsbbs.56.415 